\title{
STING-activating Cyclic Dinucleotide Agonist MIW815
}

National Cancer Institute

\section{Source}

National Cancer Institute. STING-activating Cyclic Dinucleotide Agonist MIW815. NCI

Thesaurus. Code C125902.

A synthetic, cyclic dinucleotide (CDN) and agonist of stimulator of interferon genes protein (STING; transmembrane protein 173; TMEM173), with potential immunomodulating and antineoplastic activities. Upon intratumoral administration, the STING agonist MIW815 binds to STING and stimulates STING-mediated pathways. This activates the immune response through the activation of certain immune cells, including dendritic cells (DCs), which induces the expression of cytokines and chemokines, and leads to an antigen-specific T-cell mediated immune response ag ainst cancer cells. STING, a transmembrane protein that activates immune cells in the tumor microenvironment, plays a key role in the activation of the innate immune system. 